\title{
Prospects for the spin structure study of hyperons using heavy quark decays at Belle II
}

\author{
Kiyoshi Tanida (For Belle II collaboration)* \\ Advanced Science Research Center, Japan Atomic Energy Agency \\ E-mail: tanidadpost.j-parc.ip
}

\begin{abstract}
In weak decays of heavy quarks, the daughter quarks are highly polarized. On the other hand, one can naively expect that the fraction of the baryon spin carried by a quark is equal to the polarization transfer of the quark to the baryon. In this way, one can experimentally determine the spin structures of hyperons in decays of heavy quarks. Spin structures of ground state hyperons ( i.e., $\Lambda$ and $\Sigma$ ) are interesting in the context of the proton spin structure puzzle. In addition, excited states can be also studied to identify exotic hyperons, especially $\Lambda(1405)$. With the high luminosity expected from the Belle II experiment, polarization of $\Lambda(1405)$, as well as ground $\Lambda$ and $\Sigma$, can be measured to determine its spin structure.
\end{abstract}

23rd International Spin Physics Symposium - SPIN2018 -

10-14 September, 2018

Ferrara, Italy

\footnotetext{
*Speaker.
} 


\section{Introduction}

There are lots of studies for spin structure of nucleons, and quite many things are known by now. On the other hand, spin structures of other baryons should be equally important in the view point of physics, but there are little studies so far, especially experimentally. For example, it is known that quark spin carries only about $1 / 4$ of the proton spin, and it is interesting to ask the quark spin fraction for the $\Lambda$ hyperon, of which spin is, in the quark model, solely carried by the single strange quark; however, the answer is still not available as there are very little experimental data.

Spin structure is also interesting in terms of identifying exotic baryons. For example, there is a long history of discussion on $\Lambda(1405)$, which may be a pentaquark state, a bound state of $\bar{K} N$, or just a usual three-quark state. These possibilities were not discriminated by mass spectroscopy alone, but spin structure study can give strong information. In the example of $\Lambda(1405)$, if it is a three-quark state as the naive quark model predicts, $\Lambda(1405)$ spin $(1 / 2)$ is made from the strange quark spin (1/2) and orbital angular momentum (1), so that the strange quark spin is directing to the opposite direction of the $\Lambda(1405)$ spin. On the other hand, if $\Lambda(1405)$ is a bound state of $\bar{K} N$, the strange quark is contained within the spinless $\bar{K}$ meson, and thus it cannot contribute to the $\Lambda(1405)$ spin. Therefore, if we can measure strange quark spin contribution, the structure of $\Lambda(1405)$ can be experimentally determined.

In order to study spin structure of hyperons, we use hyperon production from fragmentation of polarized quarks, which are, in turn, produced by weak decay of heavy quarks. Here, the principle is:

- In a naive picture, polarization transfer from a polarized quark to the final state baryon is equal to the fraction of spin carried by the quark [四].

Combined with

- Quarks produced by weak decay of heavy quarks are nearly $100 \%$ polarized.

- Polarization of final state hyperon can be determined by measuring its decay asymmetry.

polarization transfer from a polarized quark to the final state baryon can be measured.

In the past, there were measurements for ground state baryons $\left(\Lambda\right.$ and $\left.\Lambda_{b}\right)$ using strange (bottom) quarks produced from $Z^{0}$ decays, in which the strange (bottom) quark is also highly polarized

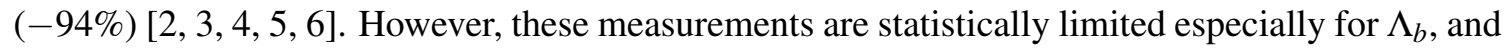
for $\Lambda_{s}$, contamination from $s \bar{s}$ pair creation during fragmentation introduced sizable uncertainty. These results are consistent with quark-model predictions within the large uncertainties.

\section{SuperKEKB and Belle II}

The Belle II experiment [ [] is a major upgrade to the Belle experiment, aiming to collect 50 times more statistics $\left(50 \mathrm{ab}^{-1}\right)$ than Belle. Such high statistics is possible thanks to the highluminosity $e^{+} e^{-}$collider, SuperKEKB [8] . A schematic diagram of SuperKEKB collider is shown in Fig. $\square$ (left). It consists of two $3 \mathrm{~km}$ rings equipped with radio-frequency (RF) systems which 
accelerate $e^{-}$and $e^{+}$beams to 4 and $7 \mathrm{GeV}$, respectively, and make them collide at the center of the Belle II detector. There are several changes with respect to the predecessor, KEKB accelerator, namely, longer dipoles and redesigned magnet lattice to squeeze the emittance, and upgraded RF systems for higher $(\times 2)$ beam currents. SuperKEKB reduces $(1 / 20)$ the beam spot size using a nano-beam scheme achieved with new superconducting final focusing quadrupole magnets near the interaction region. The upgraded collider will deliver a peak luminosity of $8 \times 10^{35} \mathrm{~cm}^{-2} \mathrm{~s}^{-1}$, which is 40 times higher than KEKB.
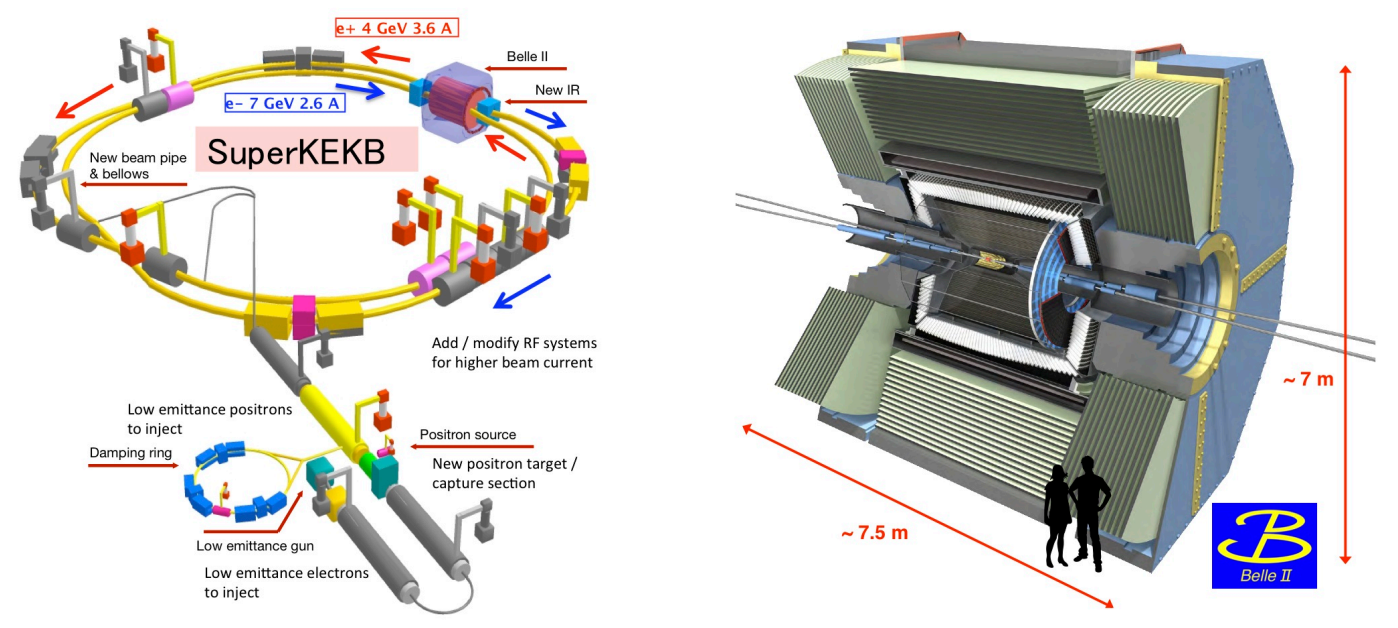

Figure 1: Schematic views of the SuperKEKB accelerator (left) and the Belle II detector (right).

The Belle II detector consists of several sub-detectors that, with respect to Belle, are either upgraded or replaced for improved detection performance at higher luminosity environment. The schematic diagram of Belle II detector is shown in Fig. W (right). A larger tracker improves the impact parameter and secondary vertex resolutions, increases the $K_{S}^{0}$ and pion effciencies, and provides better flavor tagging. A smaller beryllium double-wall beam pipe, combined with an innermost silicon pixel layer much closer to the interaction region, improve the impact parameter resolution along the beam line. An upgraded time-of-propagation (TOP) counter and ring-imaging Cherenkov counters with aerogel radiator (A-RICH), together with a faster and more hermetic $K_{L}^{0}$ and muon (KLM) detector, allow a better particle identification and further enhance the flavor tagging and background rejection. Belle II has faster and more reliable trigger and data acquisition (DAQ) systems in order to operate at a much higher event rate.

\subsection{Status of Belle II}

Operation of SuperKEKB started in 2015 (Phase 1). Phase 1 was mostly devoted for accelerator commissioning and background measurement at the Belle II collision point, and was finished in 2016. Then, Belle II (with only partial vertex detectors) was installed in 2017 and detector commissioning with cosmic rays was performed. From early 2018, Phase 2 operation was conducted with the (partial) Belle II detector. The first collision was observed on April 26th, 2018 (see Fig. $\square$ for an event display) and about $0.5 \mathrm{fb}^{-1}$ was recorded by the end of Phase 2 (July 2018). The 


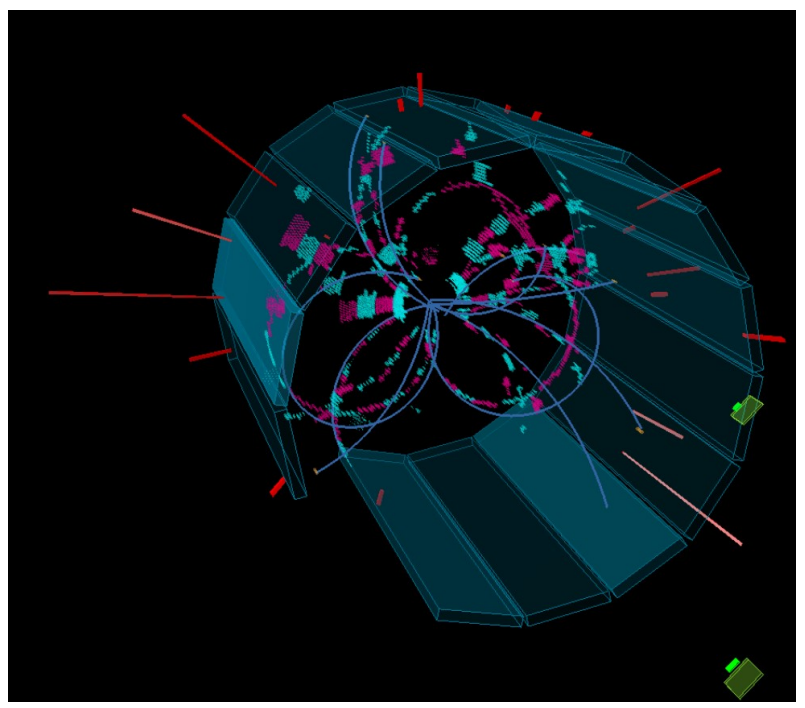

Figure 2: An event display of the first Belle II hadronic event at 0:38 am on April 26th, 2018.

peak luminosity reached to $5 \times 10^{33} \mathrm{~cm}^{-2} \mathrm{~s}^{-1}$, which is one quarter of the maximum luminosity of Belle.

Using the Phase 2 data, good detector performance is demostrated. As an example, Fig. [3] shows preliminary invariant mass plots for $K_{S}^{0} \rightarrow \pi^{+} \pi^{-}$and $\phi \rightarrow K^{-} K^{+}$. These peaks are clearly seen, demonstrating that the Belle II detector is working very well as expected and have strong capability even though most of the vertex detectors are not yet installed. Many other states are also rediscovered and the full list can be found in Ref. [Q].
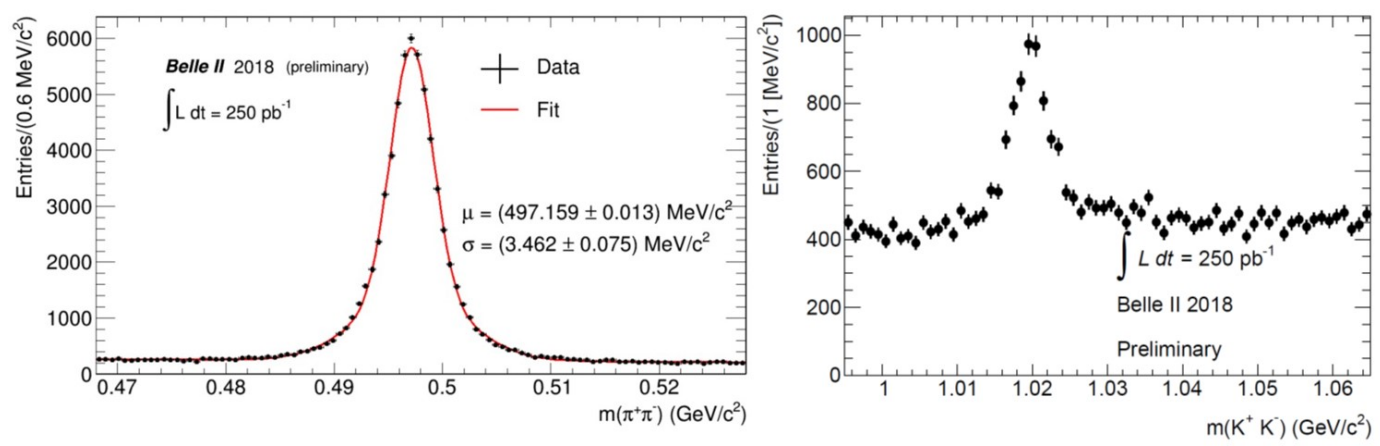

Figure 3: Invariant mass distributions for $K_{S}^{0} \rightarrow \pi^{+} \pi^{-}$(left) and $\phi \rightarrow K^{-} K^{+}$(right) candidates with 250 $\mathrm{pb}^{-1}$ of collision data.

\subsection{Near Future Operation}

We have finished installing the remaining vertex detectors in winter 2018. Then, Phase 3 operation will start from early 2019 with the full Belle II detector. We expect to reach the designed peak luminosity $\left(8 \times 10^{35} \mathrm{~cm}^{-2} \mathrm{~s}^{-1}\right)$ in 2022 , and to achieve the luminosity goal $\left(50 \mathrm{ab}^{-1}\right)$ by 2025 , as shown in Fig. 团. 


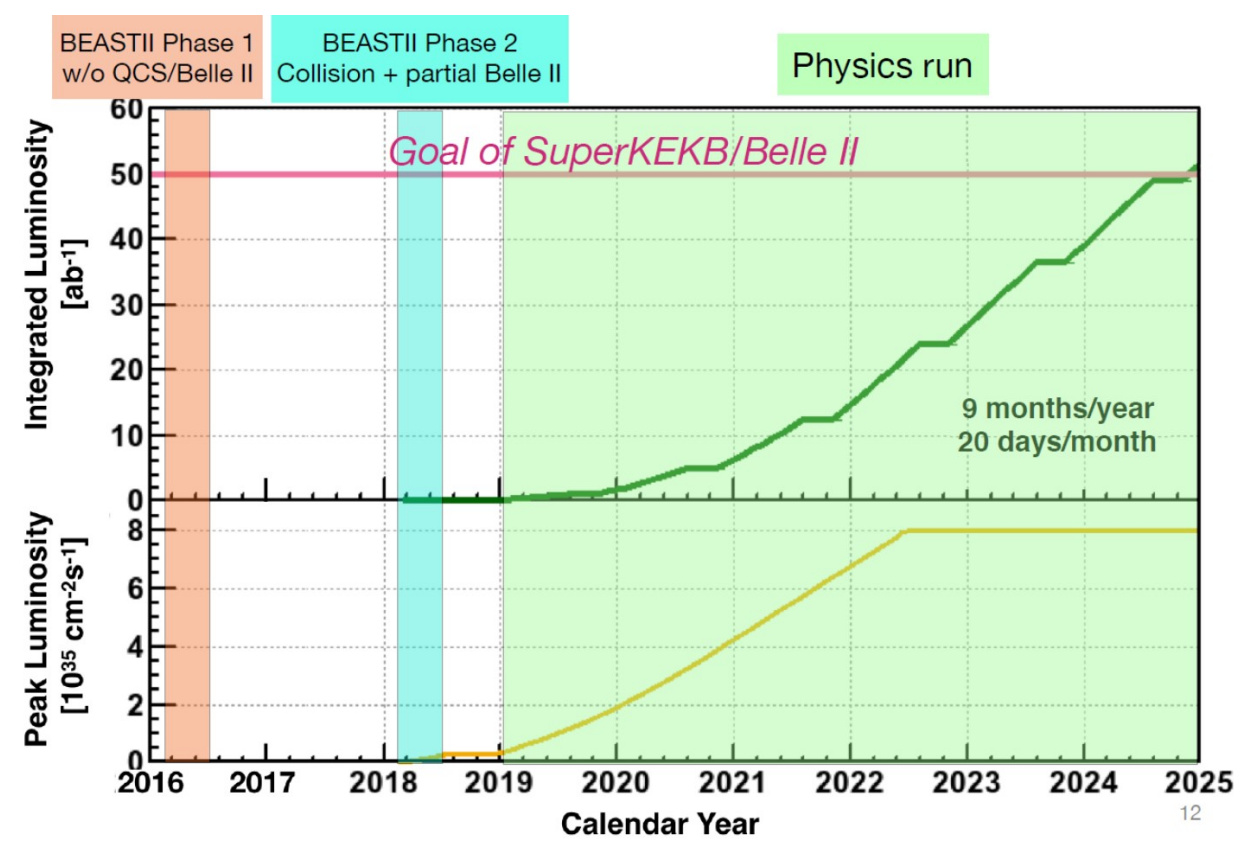

Figure 4: Luminosity projection for Belle II.

Table 1: Existing experimental data on hyperon polarization from $\Lambda_{c}$ decay (or equivalently decay asymmetry parameter $\alpha$ ).

\begin{tabular}{cc} 
decay mode & hyperon polarization [ए]] \\
\hline$\Lambda_{c} \rightarrow \Lambda e^{+}\left(\mu^{+}\right) \nu$ & $-0.86 \pm 0.04$ \\
$\Lambda_{c} \rightarrow \Lambda \pi^{+}$ & $-0.91 \pm 0.15$ \\
$\Lambda_{c} \rightarrow \Sigma^{+} \pi^{0}$ & $-0.45 \pm 0.32$ \\
\hline
\end{tabular}

\section{Possible measurements at Belle II}

Compared to the past experiments, much larger statistics is expected with $b$ and $c$ quark decays at Belle II, and studies for excited baryons will be possible. In Belle II, we will measure polarization of hyperon $Y$ in semileptonic decays $\left(\Lambda_{c} \rightarrow Y e^{+}\left(\mu^{+}\right) v\right)$ and nonleptonic decays $\left(\Lambda_{c} \rightarrow Y \pi\right)$. Polarizations of the ground state hyperons $(Y=\Lambda, \Sigma)$ would be interesting not only for their spin structure but also as control samples for excited hyperons. A few experimental data are existing for $\Lambda_{c} \rightarrow \Lambda e^{+}\left(\mu^{+}\right) \nu, \Lambda_{c} \rightarrow \Lambda \pi^{+}$, and $\Lambda_{c} \rightarrow \Sigma^{+} \pi^{0}$ and their PDG averages [U]] are summarized in Table $\mathrm{I}$. The $\Lambda$ polarizations are nearly -1 in the both decay modes, and this can be well understood from naive quark model in which the strange quark carries all the $\Lambda$ spin. The $\Sigma^{+}$case is more complicated, but anyway the uncertainty is still large to draw any conclusion. Belle II can improve the uncertainty to a few percent level, limited by systematics.

\subsection{A case for $\Lambda(1405)$}

For excited states, the most interesting case is $\Lambda(1405)$, as explained in Sect. 1. Theoreti- 
cally clean channels are semi-leptonic modes, namely, $\Lambda_{c} \rightarrow \Lambda(1405) e^{+}\left(\mu^{+}\right) v$. However, since neutrinos are missing, decay of $\Lambda_{c}$ cannot be reconstructed in invariant mass spectroscopy, and hence experimental detection is rather difficult. One way to identify $\Lambda_{c}$ production is by missing mass spectroscopy. For exmaple, in Belle, $3.6 \times 10^{4} \Lambda_{c}$ s were identified by the missing mass of $e^{+} e^{-} \rightarrow D^{(*)-} \bar{p} \pi^{+}\left(\Lambda_{c}^{+}\right)[\mathbb{W}]$. Assuming that the branching ratio to $\Lambda(1405) e^{+}\left(\mu^{+}\right) v$ is a few percent, only a few 10 counts are expected to be detected in the whole Belle data. Therefore, Belle II statistics is certainly necessary to measure $\Lambda(1405)$ polarization is semi-leptonic decays.

In contrast, non-leptonic decays are much easier experimentally, although theoretical interpretations may be difficult because of additional quarks in the final state. The decay $\Lambda_{c} \rightarrow \Sigma^{+} \pi^{+} \pi^{-}$is already observed in Belle [ㄹ] and $\Lambda(1405)$ can be searched for in the Dalitz plot. If enough statistics is obtained in Belle II, we can measure $\Lambda(1405)$ polarization as a function of $\Sigma^{+} \pi^{-}$invariant mass, and a double-pole structure suggested by Jido et al. [एँ3] may be observed.

\section{Summary}

In summary, we can experimentally approach the spin structures of hyperons in decays of $\Lambda_{c}$ using the high-statistics data expected for Belle II. Especially, $\Lambda(1405)$ polarization will be measured in semi-leptonic and non-leptonic $\Lambda_{c}$ decays to identify the nature of $\Lambda(1405)$. Doublepole strcture may appear as mass dependence of $\Lambda(1405)$ polarization.

\section{References}

[1] C. Boros, and Liang Zuo-tang, Phys. Rev. D 57, 4491 (1998).

[2] D. Buskulic et al. (ALEPH Collaboration), Phys. Lett. B 374, 319 (1996).

[3] K. Ackerstaff et al. (OPAL Collaboration), Eur. Phys. J. C 2, 49 (1998).

[4] D. Buskulic et al., (ALEPH Collaboration), Phys. Lett. B 365, 437 (1996).

[5] P. Abreu et al. (DELPHI Collaboration), Phys. Lett. B 474, 205 (2000).

[6] G. Abbiendi et al. (OPAL Collaboration), Phys. Lett. B 444, 539 (1998).

[7] T. Abe et al. (Belle-II Collaboration), arXiv:1011.0352 [physics.ins-det].

[8] Y. Morita et al., Conf. Proc. C 100523, TUPEB011 (2010).

[9] https://docs.belle2.org/collection/Belle\%20II\%20Notes\%20\%3A\%20Plots? In=en

[10] M. Tanabashi et al. (Particle Data Group), Phys. Rev. D 98, 030001 (2018).

[11] A. Zupanc et al. (Belle Collaboration), Phys. Rev. Lett. 113, 042002 (2014).

[12] M. Berger et al. (Belle Collaboration), Phys. Rev. D 98, 112006 (2018).

[13] D. Jido, J. A. Oller, E. Oset, A. Ramos and U. G. Meissner, Nucl. Phys. A 725, 181 (2003). 